\title{
Everyday postures in idiopathic scoliosis: is there any correlation with curve morphology?
}

\author{
A Negrini ${ }^{1 *}$, E Pasini ${ }^{1}$, M Romano ${ }^{1}$, S Donzelli ${ }^{1}$, F Zaina ${ }^{1}$, S Negrini ${ }^{2,3}$ \\ From 9th International Conference on Conservative Management of Spinal Deformities - SOSORT 2012 \\ Annual Meeting \\ Milan, Italy. 10-12 May 2012
}

\section{Background}

Postural control is considered important for hyperkyphosis treatment, but it has been defined of mean importance in the SOSORT Consensus on aims of idiopathic scoliosis (IS) treatment $[1,2]$. Nevertheless, no studies can be found on the usual everyday postures (UEP) of IS patients.

\section{Aim}

To verify if IS patients adopt specific asymmetric UEP.

\section{Methods}

Through parents and scoliosis experts' consultation, we developed and validated a questionnaire for parents evaluating 7 UEP. Inclusion criteria were IS and age between 6 and 18 years. We collected 635 questionnaires from all IS patients coming to our Institute between September and November $2011(\mathrm{n}=435$, response rate 98.5\%) and through specific emails $(n=199$, response rate $15.7 \%)$. Since there were no differences between these two groups in gender, age, scoliosis parameters and answers, we evaluated all questionnaires together. We had IS group (ISG: curves $>10^{\circ} ; \mathrm{n}=462$ ) and controls (CG: curves $<10^{\circ} ; \mathrm{n}=173$ ). We divided ISG into three pairs of subgroups:

L: lumbar or thoraco-lumbar curve: left (LL-SG $n=65)$; right (RL-SG $\mathrm{n}=56$ )

$\mathrm{T}$ : thoracic curve: right (RT-SG $\mathrm{n}=79$ ); left (LT-SG $\mathrm{n}=11$ )

DC: double curves: left $\mathrm{L}$ right $\mathrm{T}$ (LRDC-SG: $\mathrm{n}=215$ ); right $\mathrm{L}$ left $\mathrm{T}$ (RLDC-SG $\mathrm{n}=36$ )

We compared ISG and all subgroups to CG, and each subgroup to its matched pair (e.g. LL-SG vs RL-SG). All answers were converted as follows: one side: +1 ; the other side: -1 ; no preference: 0 . Maintaining only the UEP with statistical differences, and checking for the preferred

${ }^{1}$ ISICO (Italian Scientific Spine Institute), Milan, Italy

Full list of author information is available at the end of the article direction, we developed three index of symmetry (IoS) (one per pair of subgroups). Finally, we checked correlations between Cobb degrees, UEPs and IoS.

\section{Results}

In L subgroups, we found one UEP statistically different between the matched pairs and 2 from CG; in the T subgroups the differences were 2 and 2 respectively; in the DC subgroups, only LRDC-SG had 3 postures different from CG. The calculated IoS were significantly different in the the $\mathrm{L}$ and $\mathrm{T}$ subgroups, but not in $\mathrm{DC}$. There were no statistical correlations with Cobb degrees.

\section{Conclusions}

IS patients have preferred UEP, mainly in the case of single curves; postural control strategies should be considered in future rehabilitation protocols.

\section{Author details \\ ${ }^{1}$ ISICO (Italian Scientific Spine Institute), Milan, Italy. ${ }^{2}$ University of Brescia, Brescia, Italy. ${ }^{3}$ IRCCS Don Gnocchi, Milan, Italy.}

Published: 3 June 2013

\section{References}

1. Weiss HR, Negrini S, Hawes MC, Rigo M, Kotwicki T, Grivas TB, Maruyama T: Physical exercises in the treatment of idiopathic scoliosis at risk of brace treatment - SOSORT consensus paper 2005. Scoliosis 2006, 1:6.

2. Negrini S, Grivas TB, Kotwicki T, Maruyama T, Rigo M, Weiss HR: Why do we treat adolescent idiopathic scoliosis? What we want to obtain and to avoid for our patients. SOSORT 2005 Consensus paper. Scoliosis 2006, 1:4.

3. Fusco C, Zaina F, Atanasio S, Romano M, Negrini A, Negrini S: Physical exercises in the treatment of adolescent idiopathic scoliosis: an updated systematic review. Physiother Theory Pract 2011, 27(1):80-114.

\section{doi:10.1186/1748-7161-8-S1-06}

Cite this article as: Negrini et al:: Everyday postures in idiopathic scoliosis: is there any correlation with curve morphology? Scoliosis 2013 8(Suppl 1):06. 